\title{
Nuclear import of the human androgen receptor
}

\author{
Guido JENSTER, ${ }^{\star} \dagger$ Jan TRAPMAN $\ddagger$ and Albert 0. BRINKMANN* \\ Department of ${ }^{*}$ Endocrinology and Reproduction and $\ddagger$ Department of Pathology, Erasmus University, P.0. Box 1738, 3000 DR Rotterdam, The Netherlands
}

Nuclear import of the human androgen receptor was investigated by immunocytochemical analysis of androgen receptor deletion and substitution mutants, which were transiently expressed in COS-1 cells. The signal responsible for nuclear import is encoded by amino-acid residues $608-625$ and is functionally similar to the bipartite nucleoplasmin nuclear-localization signal. Although the subcellular distribution of androgen receptors mutated in the DNA-binding domain was unchanged compared with the wildtype androgen receptor, in the presence of ligand these mutations resulted in part of the receptor population forming clusters. Depending on the presence or absence of the bipartite nuclear localization signal, clusters were formed in the nucleus or in the cytoplasm, respectively. Expression of the wild-type androgen receptor in different cell lines revealed a cell-line-specific subcellular distribution of the unliganded receptor. The androgen receptor was predominantly nuclear when expressed in $\mathrm{HeLa}$ cells, whereas mainly cytoplasmic staining was observed when it was expressed in COS- 1 cells. In the presence of hormone, the androgen receptor was located in the nucleus, independent of the cell line that was expressing the receptor. Anti-androgens and various steroid hormones induced the nuclear localization of the wild-type androgen receptor in a dose-dependent way, without activating transcription of an androgen-regulated reporter gene. This indicates that the inability of the tested compounds to activate transcription is not due to inhibited nuclear import.

\section{INTRODUCTION}

The androgen receptor (AR) is a ligand-dependent transcription factor that belongs to the large family of steroid-hormone receptors (Evans, 1988; Green and Chambon, 1988). Activated by their ligand, these receptors interact with specific DNA sequences located in the $5^{\prime}$ flanking regions of target genes, resulting in the up- or downregulation of the expression of these genes. Before exerting their function as transcription factors, these proteins have to pass through the nuclear membrane. Two processes for nuclear import of proteins are commonly considered (Nigg et al., 1991; Silver, 1991). Proteins either freely diffuse through the nuclear membrane or harbour a nuclear-localization signal (NLS) that results in active transport. For passive diffusion through nuclear pores, proteins must not exceed the apparent exclusion limit of approx. $60 \mathrm{kDa}$ (Peters, 1986). Larger proteins, such as the human $A R$, need specific signals for active nuclear import.

The subcellular localization of the unliganded progesterone (PR), oestradiol (OR) and glucocorticoid (GR) receptors is still a point of discussion (Perrot-Applanat et al., 1985; Sanchez et al., 1990; Power et al., 1991 ; Brink et al., 1992). Contradictory findings have been published for the $A R$ as well. Immunohistochemical studies on the subcellular localization of the AR in normal and castrated rats demonstrated that the AR was located in the nucleus (Husmann et al., 1990; Sar et al., 1990), as is the case in comparable studies on prostate tissue from prostate cancer patients under androgen-ablation therapy (van der Kwast et al., 1991). In contrast, the AR transiently overexpressed in COS cells was found to be localized in the cytoplasm (Simental et al., 1991) or distributed over the cytoplasm and the nucleus (Jenster et al., 1991) in the absence of ligand, and was found to be exclusively nuclear only in the presence of androgen.

One of the best characterized NLSs is the nuclear targeting signal of simian virus 40 (SV40) large $\mathrm{T}$ antigen, which consists of seven amino-acid residues, of which five are positively charged
(Kalderon et al., 1984a,b; Lanford and Butel, 1984). A similar basic signal is also present in the hinge region of all steroid receptors and its importance for nuclear import has been determined for the PR, OR, GR and the AR (Picard and Yamamoto, 1987; Guiochon-Mantel et al., 1989; Simental et al., 1991; Ylikomi et al., 1992). Deletion of this SV40 large T antigen-like NLS, however, did not result in the exclusive cytoplasmic localization of the PR and AR in the presence of hormone, indicating the complexity of the NLS and/or the presence of additional signals.

In the nuclear protein nucleoplasmin, an NLS has been identified that consists of 16 amino-acid residues, which includes a basic lysine sequence similar to the SV40 large T antigen NLS. Two basic amino-acid residues (Lys, Arg) followed by a spacer of ten residues precede the lysine stretch, forming in this way a bipartite NLS (Robbins et al., 1991). This conserved, bipartite NLS is present in many other nuclear proteins, including steroid receptors (Dingwall and Laskey, 1991).

To identify the position of possible NLSs and to define in detail the contribution of the bipartite nucleoplasmin-like NLS to AR nuclear import, we have constructed a series of mutants of the human AR and have immunocytochemically examined the subcellular localization of these proteins. In addition, the effect of anti-androgens and various steroid hormones on the nuclear import of the AR was investigated.

\section{MATERIALS AND METHODS \\ Materials}

R1881 (17 $\alpha$-methyltrienolone) and R5020 (promegestone) were purchased from NEN (Boston, MA). Cyproterone acetate was a gift from Schering (Berlin, FRG), hydroxyflutamide was from Schering U.S.A. (Bloomfield, NJ) and ICI 176334 (trademark 'Casodex') was from ICI Pharmaceuticals (Macclesfield, 
Cheshire, U.K.). All other steroids were purchased from Steraloids (Wilton, NH, U.S.A.).

\section{Cell culture, transfection and chloramphenicol-acetyltransforase assay}

COS-1, HeLa, CV-1 and Chinese hamster ovary (CHO) cells were maintained in Eagle's minimum essential medium supplemented with $5 \%$ fetal-calf serum, non-essential amino acids and antibiotics. The cells were transfected using the calciumphosphate precipitation method (Chen and Okayama, 1987). AR functional activity was assessed in COS-1 cells by transient transfection as described previously (Jenster et al., 1991). The reporter plasmid contains two progesterone-responsive/glucocorticoid-responsive elements (PRE/GREs) in front of the thymidine kinase (tk) promoter linked to the chloramphenicol acetyltransferase (CAT) gene (pG29GtkCAT; Schüle et al., 1988). CAT assays representative of at least three independent experiments are shown.

\section{AR expression vectors}

A human AR cDNA expression vector (pAR0) was constructed using the SV40 early promoter and the rabbit $\beta$-globin polyadenylation signal (Brinkmann et al., 1989). Construction of the AR mutants pAR6, pAR7, pAR13, pAR14, pAR17 and pAR21 has been described (Jenster et al., 1991). The mutant pAR12 is a C-terminal truncated mutant, made by introduction of a stop codon into the AR cDNA. For this purpose an $X b a I$ linker (Promega, Madison, WI, U.S.A.) that contains an in-frame stop codon was ligated into the blunt-ended Asp 718 site (Jenster et al., 1991). The mutant pAR4 was constructed from an aberrant cDNA clone that lacks exons 4-8. The first part of intron 3 is present so that translation will result in a protein encoded by exons 1,2 and 3 and 20 additional amino-acid residues encoded by intron 3 (residues 618-638: GGKILFFLFLLLLLSPFSLIF). Deletion mutants pAR61 and pAR63 were constructed by removal of internal fragments using two different restriction enzymes. The combination of the restriction enzymes was chosen such that ligation of the blunt-ended plasmid would result in an in-frame deletion. The restriction enzymes $\mathrm{NruI}$ and $A s p 718$ were used to construct pAR61, and Asp718 and SacI were used to construct pAR63 (Jenster et al., 1991). The mutant pAR8 was made using the pSTCGR3-795 vector, containing the cytomegalovirus (CMV) promoter and the rabbit $\beta$-globin polyadenylation signal (Severne et al., 1988), which was digested with BamHI to remove the rat GR cDNA, and was made blunt-ended. An AR cDNA fragment was isolated from pAR0 by SmaI digestion and was inserted into the pSTC vector. The mutants pAR22, pAR64, pAR65 and pAR28.X were constructed by sitedirected mutagenesis, using PCR DNA amplification techniques (Higuchi et al., 1988). The following primers, introducing the deletion or substitution, were used: in COS-1 cells and the protein produced was analysed by Western blotting (Jenster et al., 1991).

\section{Immunocytochemistry}

COS-1, HeLa, CV-1 and CHO cells were cultured directly on microscope glass slides $\left(2 \times 10^{5}\right.$ cells/slide $)$ and were transfected using the calcium-phosphate method ( $2 \mu \mathrm{g}$ of AR plasmid and $5 \mu \mathrm{g}$ of pTZ19 carrier plasmid/slide). The cells were plated and transfected in medium containing $5 \%(\mathrm{w} / \mathrm{v})$ dextran/charcoaltreated fetal-calf serum. Fixation and permeabilization of the transfected cells was performed essentially as described previously (Jenster et al., 1991). After the permeabilization the cells were rehydrated in PBS and incubated for $15 \mathrm{~min}$ in PBS containing $10 \%(\mathrm{v} / \mathrm{v})$ fetal-calf serum. Slides were incubated with monoclonal antibody F39.4.1, diluted 1:1000 (Zegers et al., 1991) or polyclonal antibody Sp197, in PBS containing $1 \%(w / v)$ BSA for $1 \mathrm{~h}$ at room temperature. Excess antibody was removed by washing, followed by a $1 \mathrm{~h}$ incubation with fluorescein-isothiocyanate (FITC)- or tetramethyl rhodamine B isothiocyanate (TRITC)-conjugated goat anti-mouse or goat anti-rabbit immunoglobulin (Sigma, St. Louis, MO, U.S.A.), diluted $1: 1000$ in PBS containing $1 \%(\mathrm{w} / \mathrm{v})$ BSA. After several PBS washes, slides were mounted with $90 \%(\mathrm{v} / \mathrm{v})$ glycerol in PBS. The polyclonal antibody Sp197 (designed according to Husmann et al., 1990) contains a high titre of AR antibodies, as was shown by immunoprecipitation and Western blotting. F39.4.1 and Sp197 antibody specificity has been determined by mock transfection of COS-1, HeLa, CV-1 and CHO cells, showing background staining only. AR subcellular localization was classified into five different categories $(\mathrm{N} ; \mathrm{N}>\mathrm{C} ; \mathrm{C}=\mathrm{N} ; \mathrm{C}>\mathrm{N} ; \mathrm{C}$ ) based upon intensity of fluorescence in the cytoplasm (C) compared to the nuclear $(\mathrm{N})$ fluorescence intensity. In the case where the distribution is $\mathrm{C}=\mathrm{N}$, the fluorescence intensity is the same in both compartments. In each transfection experiment at least 50 positive cells were scored. The category that was scored the most is the one that is reported. In almost all cases more than $75 \%$ of the positive cells were classified into the same category. The transfection experiments were performed at least three times.

\section{RESULTS}

\section{Cell-line-specific subcellular localization of the human AR}

One of the possible explanations for the contradictory reports on the subcellular localization of the unliganded AR may be the use of different cell lines in the various studies. To investigate the subcellular localization in different cell lines, COS-1, HeLa, CV-1 and CHO cells were transiently transfected with the pARO expression vector coding for the wild-type human AR (Brinkmann et al., 1989). As shown in Figure 1, in the absence of

\section{pAR22:5' GCAGTGTCGGTATCGATGGACTACTACAACTTTCCA 3' pAR64: 5' GCTCTCACATCTGGAAGCTTCAAGGTCTTC 3' pAR65:5' CCGAAGGAAGAATTCTCCATCTTCTCGTC 3' pAR28.1:5' GGATGACTCTGGGCGCCGGCAATCTGAAACTACAG 3' pAR28.2: $5^{\prime}$ AGCAGGGATGACTCTGGGCATGCTGAAACTACAGGAGGAAG 3' pAR28.3:5' TGTCCATCTTGTCGTCTTGGCGCCTGTTATGAAGCAGGGATG 3'}

The mutant pAR28.23 is a combination of mutants pAR28.2 and pAR28.3. The mutant pAR28.31 is a combination of pAR28.1 and pAR28.3. All the mutants were sequenced to verify the correct reading frame. In addition, the mutants were expressed ligand, the AR in COS-1 cells was predominantly cytoplasmic, whereas in CV-1 and CHO cells, the AR was more evenly distributed over the cytoplasm and nucleus. In HeLa cells, 


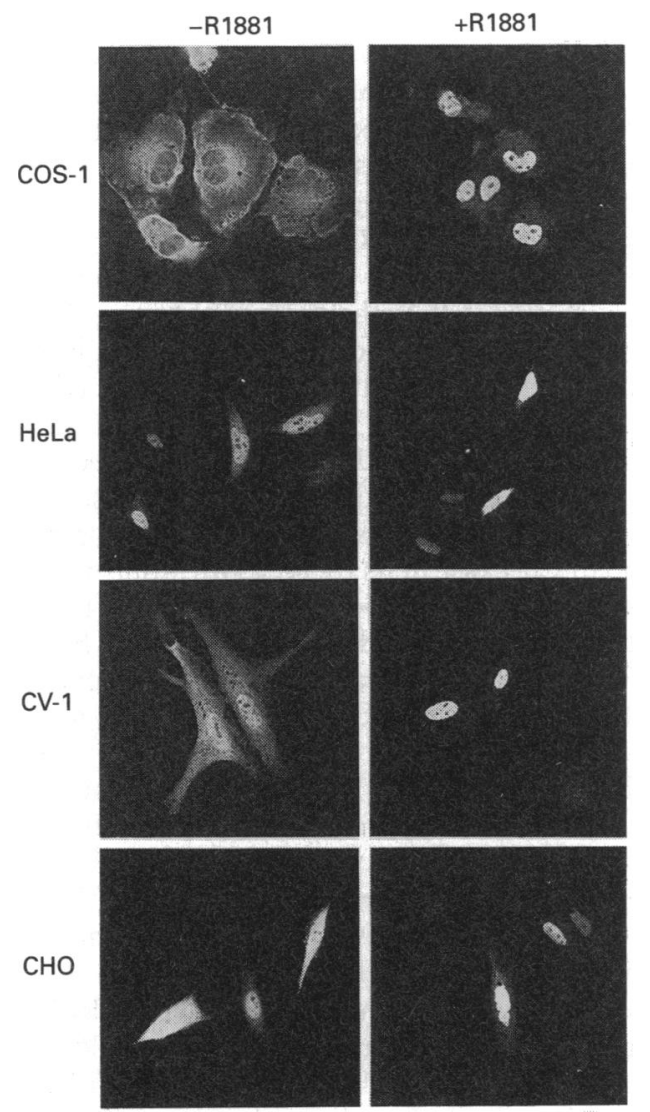

Figure 1 Immunocytochemical localization of the wild-type AR in different cell lines

COS-1, HeLa, CV-1 and CHO cells were plated on to microscope slides and transfected using the calcium-phosphate method. One day after the addition of the DNA precipitate, the cells were washed and incubated for $24 \mathrm{~h}$ either with $10 \mathrm{nM}$ R1881 (+R1881) or without (-R1881). Subsequently, the cells were fixed and stained using the F39.4.1 monoclonal antibody and fluorescein-isothiocyanate-coupled goat anti-mouse antibodies, as described in the Materials and methods section. Magnification is approx. $250 \times$.

unliganded AR was predominantly nuclear, indicating that the subcellular distribution is indeed cell-line specific. Addition of $10 \mathrm{nM}$ of the synthetic androgen R1881 resulted in the nuclear localization of the AR in all cell lines (Figure 1).

\section{Subcellular locallzation of AR deletion mutants}

To identify the position of possible NLSs, AR deletion mutants were transiently expressed in COS- 1 cells and their subcellular localization was examined. The results of these experiments are summarized in Figure 2.

The deletion of part of the steroid-binding domain (AR17) resulted in a receptor that was mainly located in the cytoplasm. This protein is unable to bind ligand and therefore the addition of $10 \mathrm{nM}$ R1881 had no effect. AR mutants lacking even larger parts of the C-terminal domain (AR13 and AR14) were exclusively nuclear, indicating that a region present in AR17, but deleted in AR14 (amino-acid residues 713-796) is responsible for the cytoplasmic retention. Both AR13 and AR14 show constitutive transactivation activity (Jenster et al., 1991). Deletion up to amino-acid residue 618 results in a truncated receptor (AR4) that was mainly cytoplasmic. This observation suggests the presence of an NLS between residues 618 and 653. An AR

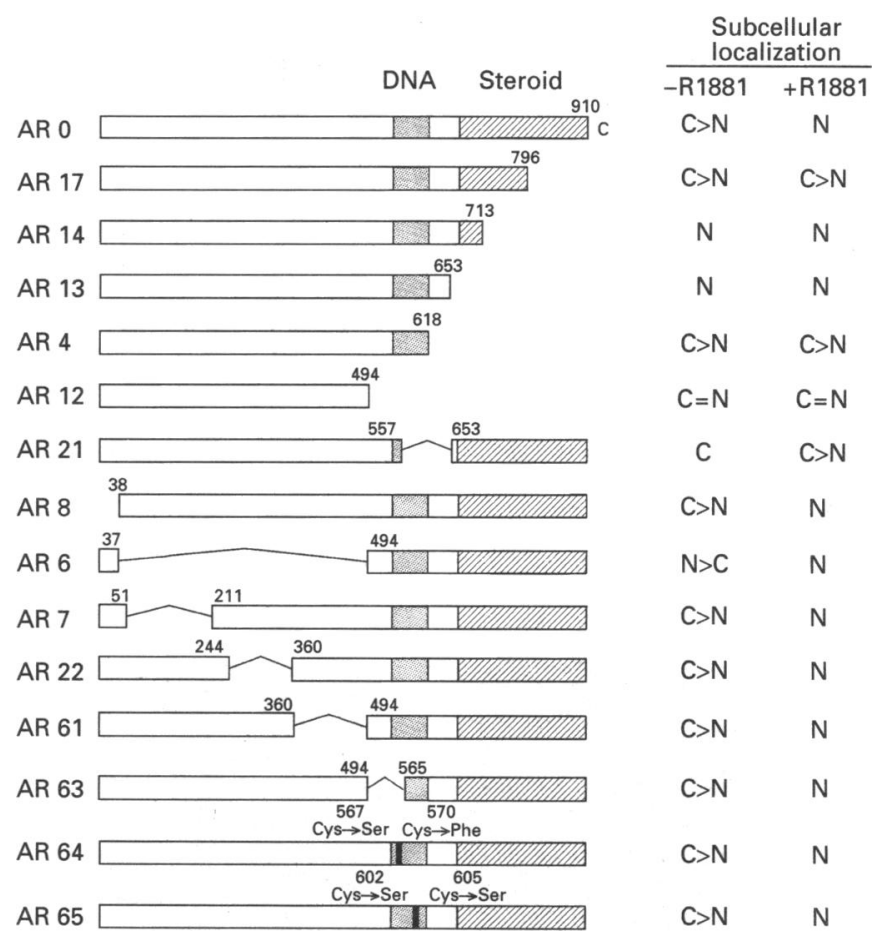

Figure 2 Subcellular localization of human AR mutants

Nuclear and cytoplasmic localization of AR proteins expressed in COS-1 cells in the absence (-R1881) or presence (+R1881) of $10 \mathrm{nM}$ R1881. The receptor mutants were visualized using the F39.4.1 antibody, except for AR22, for which the Sp197 antibody was used.
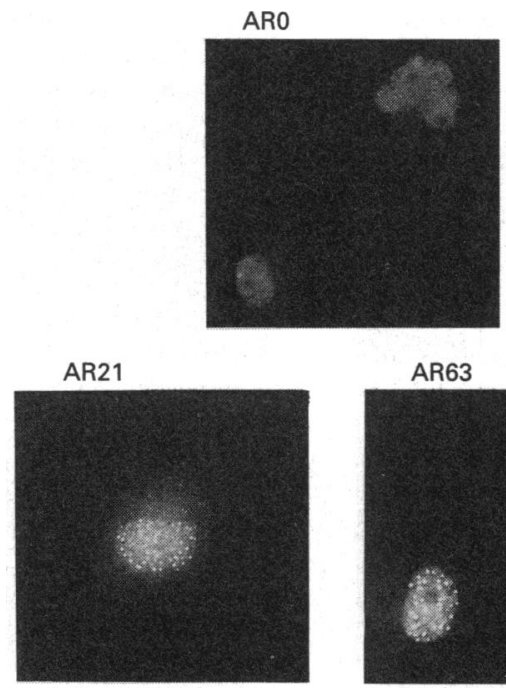

AR63
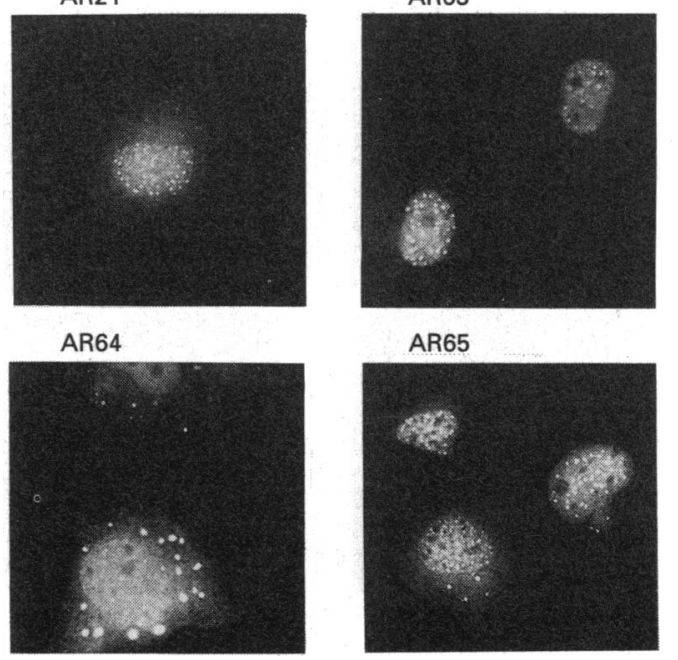

Figure 3 staining pattern of the wild-type AR (ARO) and of AR mutants expressed in COS-1 cells in the presence of $10 \mathrm{nM}$ R1881

The receptors were visualized using the F39.4.1 antibody. Magnification is approx. $630 \times$. 

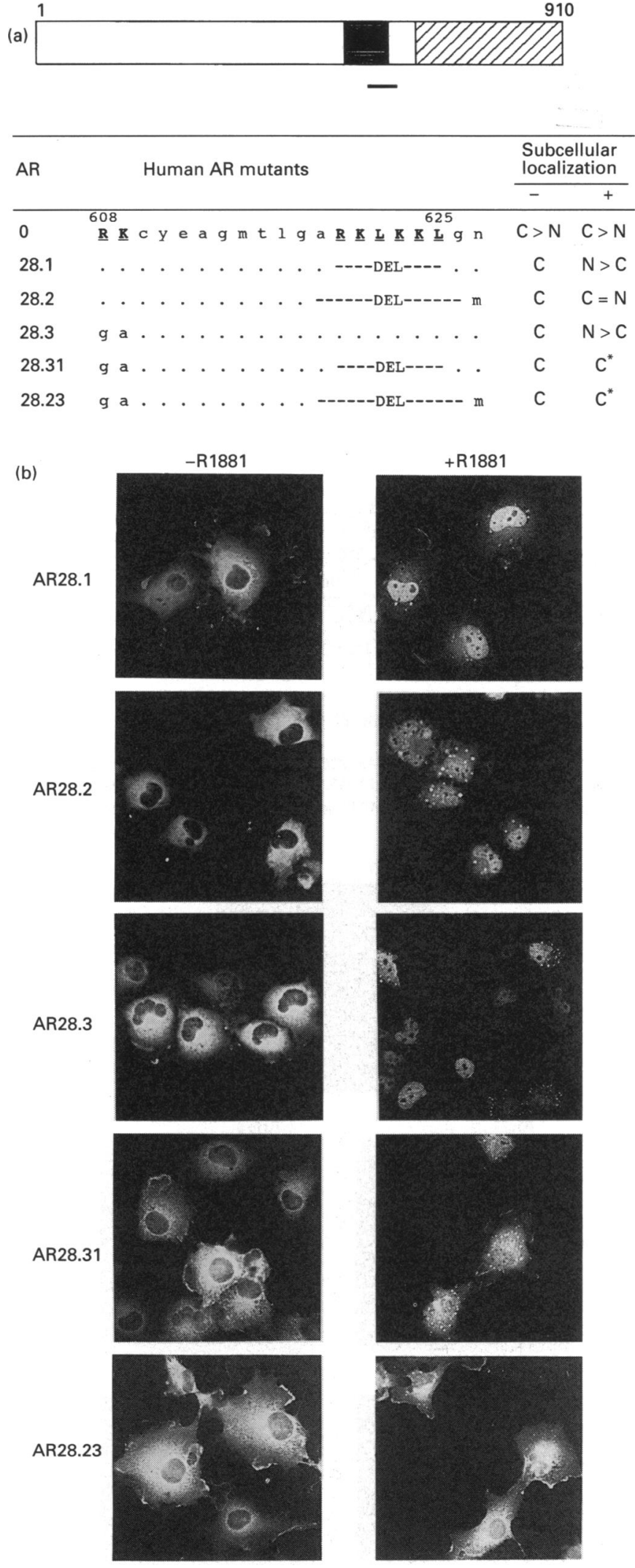

Figure 4 Mutational analysis of the nucleoplasmin-like nuclear localization signal in the wild-type AR

(a) The wild-type AR, with the NLS shown as a bar. The NLS is given below in single letter code (amino-acid residues 608-627). Dots represent unchanged residues, deletions are represented as lines with the word 'DEL' and the newly encoded residue is shown in case of mutant, lacking both the steroid-binding domain and the DNAbinding domain (AR12), was evenly distributed over the cytoplasm and nucleus. This truncated protein, with a molecular mass of approx. $60 \mathrm{kDa}$, is probably small enough to passively diffuse through the nuclear pores.

The second indication for the position of an NLS came from the subcellular localization of AR21, which lacks the amino-acid residues 557-653 (including part of the DNA-binding domain). We previously reported that, in the absence of ligand, AR21 was exclusively cytoplasmic; the addition of hormone directed a portion of the receptor population to the nucleus (Figure 2; Jenster et al., 1991).

Deletions in the $\mathrm{N}$-terminal domain did not affect the AR nuclear import. Unliganded AR6, which lacks almost the entire $\mathrm{N}$-terminal part, had a more nuclear than cytoplasmic distribution. Probably because of its reduced size (approx. $53 \mathrm{kDa}$ ), this AR mutant was able to passively diffuse through the nuclear membrane. The subcellular localizations of AR8, AR7, AR22, AR61 and AR63, all of which lack part of the N-terminal domain, were similar to the wild-type AR distribution. AR64 and AR65 were also similarly distributed, compared with the AR0 subcellular distribution (Figure 2). In these receptor mutants, the structure of the first (AR64) or second (AR65) zinc twist is disrupted by the replacement of two of the four cysteine residues that are involved in the co-ordination binding of the zinc atoms. This indicates that effective DNA binding is not involved in the subcellular distribution of the AR.

It was observed that AR21, AR63, AR64 and AR65 gave a different staining pattern in comparison with the wild-type AR staining. In contrast with the diffuse granular distribution of AR0 in the cytoplasm or nucleus, large clusters of AR21 protein were visible in the cytoplasm of most transfected cells in the presence of hormone (Figure 3). Ligand-induced clusterformation was also observed for part of the receptor population of AR63, AR64 and AR65. The AR63, AR64 and AR65 clusters, however, were mainly located in the nucleus (Figure 3 ). These findings indicate that the disruption of the DNA-binding domain results in clustering of the mutated $A R$ and that clustering is independent of the subcellular localization.

\section{Definition of an AR NLS}

From the studies with the mutants AR4 and AR21, it was concluded that amino-acid residues 557-653 are necessary for proper nuclear import. This part of the AR harbours at position 620-624 the sequence RKLKK, which is an SV40 large T antigen NLS-like sequence. This signal can partially account for the nuclear import of the AR, PR and OR (Guiochon-Mantel et al., 1989; Simental et al., 1991; Ylikomi et al., 1992). The same region also shares sequence similarity with the nucleoplasmin NLS, which consists of two basic residues, a spacer of ten aminoacid residues and a C-terminal basic domain, similar to the SV40 large $\mathrm{T}$ antigen NLS (Table 1). In nucleoplasmin, both basic parts are involved in nuclear targeting (Robbins et al., 1991). The NLS of nucleoplasmin is conserved among steroid receptors. To investigate if this bipartite NLS can account for the total nuclear

amino-acid substitutions. AR mutant numbers are on the left and the subcellular localization in the absence $(-)$ or in the presence $(+)$ of $10 \mathrm{nM}$ R1881 is shown on the right, as cytoplasmic, nuclear or combinations of cytoplasmic and nuclear staining. AR28.31 and AR28.23 showed only a very faint nuclear staining in the presence of ligand, indicated as $C^{*}$. Basic residues thought to be important for the function of the signal are in bold underlined capitals. (b) Immunofluorescence images of the AR mutants expressed in COS-1 cells in the absence (-R1881) or in the presence (+R1881) of $10 \mathrm{nM}$ R1881. Receptor mutants were visualized using the F39.4.1 monoclonal antibody. 
Table 1 Comparison of the amino-acid sequences of proven or potential NLSs in SV40 large T antigen, nucleoplasmin and various sterold-hormone receptors

The numbers refer to the first amino-acid residue of the sequence. Basic amino-acid residues thought to be important for the signal are shown in bold capitals.

\begin{tabular}{|c|c|c|}
\hline Protein & Organism & Sequence \\
\hline SV40 large $T$ antigen & SV40 & pKarKRK \\
\hline Nucleoplasmin & Xenopus & 152 kavKRpaatkkagqaKuরRl \\
\hline AR & Human & 605 crlRKcyeagmtlgaRKIRKl \\
\hline PR & Human & 622 crlRKccqagmvlggRRfKRf \\
\hline GR & Human & 476 cryRKclqagmn leaRRt KRKk \\
\hline $\begin{array}{l}\text { Mineralocorticoid } \\
\text { receptor }\end{array}$ & Human & 658 crlqKclqagmnlgaRRsKRl \\
\hline OR & Human & 240 crlRKcyevgmmkggiRKdRRg \\
\hline
\end{tabular}

import of the AR, both basic parts were either separately or simultaneously mutated.

As shown in Figures 4(a) and 4(b), mutation of either basic domain affected nuclear import (AR28.1, AR28.2 and AR28.3). In the presence of ligand, however, part of the receptor population was still nuclear. Simultaneous mutation of both basic domains (AR28.31 and AR28.23) resulted in AR mutants that were cytoplasmic in the absence of ligand, and were predominantly cytoplasmic with only a faint nuclear staining in the presence of $1 \mathrm{nM}$ R1881 (Figures $4 \mathrm{a}$ and $4 \mathrm{~b}$ ). The mutations introduced in the NLS of these AR mutants, in particular the replacement of Arg-Lys by Gly-Ala in the first basic domain of the NLS, resulted in cluster formation, again indicating the role of the DNA-binding domain in receptor clustering (Figure 4b).

The same set of NLS mutations were introduced into the constitutively nuclear AR13, which lacks the steroid-binding domain. Again, alteration of either one of the basic domains hindered nuclear import. Simultaneous modification of both basic domains in AR13 clearly affected nuclear transport; however, a small portion of the receptor population was still nuclear. The subcellular localization of these mutants derived from AR13 was investigated, both in the absence and in the presence of ligand. As expected, hormone had no effect on the distribution (results not shown).

\section{Hormone speclficity of AR nuclear import}

Next, the ability of anti-androgens and of various steroids to induce nuclear import of the AR was investigated. COS-1 cells expressing the wild-type AR were incubated for $2 \mathrm{~h}$ with different concentrations $(1000,100,10,1$ and $0.1 \mathrm{nM})$ of R5020 (promegestone), oestradiol and dexamethasone, or the anti-androgens

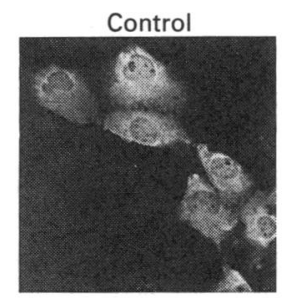

$1 \mathrm{nM}$ R1881

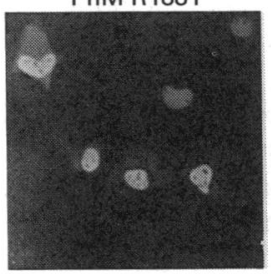

$1 \mathrm{nM}$ CA

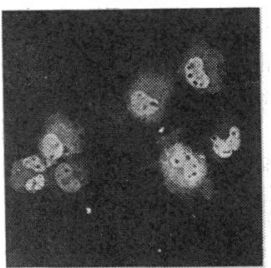

$100 \mathrm{nM} \mathrm{OH}$-flutamide

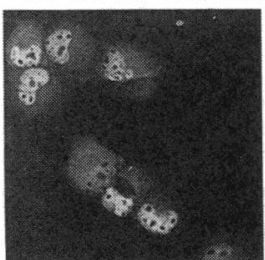

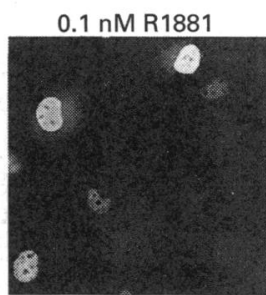

1 nM R5020

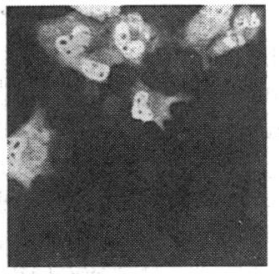

$100 \mathrm{nM}$ Casodex

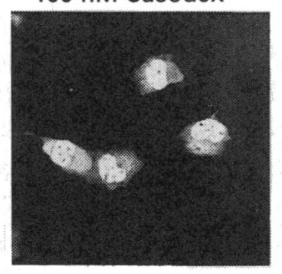

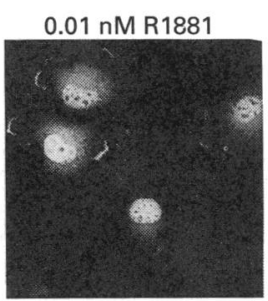

$1 \mathrm{nM}$ E2

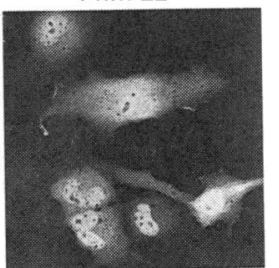

$100 \mathrm{nM}$ Dex

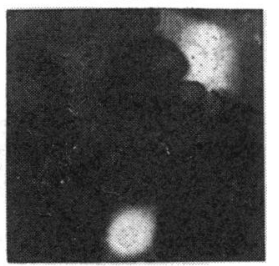

Figure 5 Subcellular localization of the wild-type AR in the absence (control) or in the presence of varlous sterold hormones and anti-androgens

COS-1 cells that had been transfected with pSVARO were incubated with different concentrations from 0.01 to $1000 \mathrm{nM}$ of steroid or of anti-androgens for $2 \mathrm{~h}$ before fixation. Immunofluorescence images are shown of the lowest concentration of steroids or anti-androgens that resulted in nuclear retention. The wild-type AR was visualized using the F39.4.1 antibody. Abbreviations used: CA, cyproterone acetate; E2, oestradiol; $\mathrm{OH}$-flutamide, hydroxyflutamide; dex, dexamethasone. 

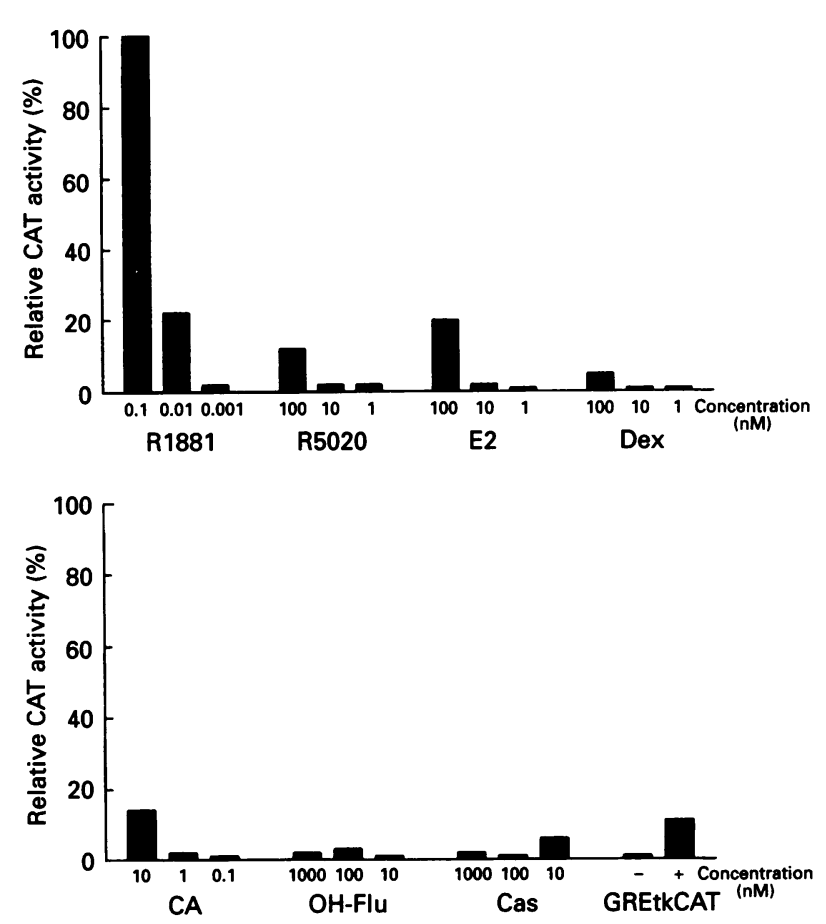

Figure 6 Transcription activation of the wild-type AR induced by various steroid hormones and anti-androgens

CAT activity was determined in COS-1 cells transfected with PSVARO and the PG29GtkCAT reporter plasmid (Schüle et al., 1988). One day before cell lysis, cells were incubated with different concentrations of R1881, R5020, oestradiol (E2), dexamethasone (Dex), cyproterone acetate (CA), hydroxyflutamide (OH-Flu) and Casodex (Cas) as indicated. Transfection of only the reporter construct is shown as GREtkCAT in the absence of hormone $(-)$ or in the presence of $0.1 \mathrm{nM} \mathrm{R1881}(+)$. Activities were corrected for the assay background that was determined by mock transfection, and are presented as a percentage relative to that of $0.1 \mathrm{nM} R 1881$ induction.

hydroxyflutamide, Casodex (ICI 176334) and cyproterone acetate. All compounds were capable of directing the AR to the nucleus, although concentrations of more than $100 \mathrm{nM}$ hydroxyflutamide, Casodex or dexamethasone and of more than $1 \mathrm{nM}$ cyproterone acetate, R5020 or oestradiol were necessary (Figure 5 ). None of the compounds tested, however, induced transcriptional activation of an androgen-regulated reporter gene (GREtkCAT) at concentrations that already resulted in a shift of the AR to the nucleus (Figure 6). This indicates that the inability of the tested compounds to induce transactivation is not due to the impairment of nuclear import.

\section{DISCUSSION}

NLSs are essential for the transport of large proteins, such as the AR, into the nucleus (Nigg et al., 1991; Silver, 1991). In previous publications on the nuclear import of the GR, PR and AR, a candidate signal similar to the SV40 large $T$ antigen NLS has been described (Picard and Yamamoto, 1987; Guiochon-Mantel et al., 1989; Simental et al., 1991). Deletion of this signal, however, does not always prevent nuclear import, indicating the complexity of the signal and/or the presence of additional signals. In the present study we show that the nucleoplasmin-like NLS, partially overlapping the SV40 large T antigen-like NLS, can account for nuclear import of the human AR. This nucleoplasmin NLS, composed of two basic parts separated by a spacer of ten amino-acid residues, is highly conserved among steroid receptors and appears to be present in many nuclear proteins (Dingwall and Laskey, 1991; Robbins et al., 1991). Mutational analysis of the bipartite nucleoplasmin-like NLS revealed that both basic parts contribute to the nuclear targeting of the AR. Substitution of two amino-acid residues in the first basic half of the NLS or deletion of the second basic domain clearly affected nuclear import, but did not result in the exclusive cytoplasmic localization of these AR mutants. Mutation of both NLS parts resulted in an AR mutant that was unable to enter the nucleus; only a very faint staining of the nucleus was observed. For the GR it has been shown that the steroid-binding domain is also capable of directing a $\beta$-galactosidase fusion protein to the nucleus (Picard and Yamamoto, 1987), indicating the presence of an additional NLS in this domain. There is no evidence for additional NLSs in the AR apart from the faint nuclear staining of AR mutants with the nucleoplasmin-like NLS deleted (AR28.23 and AR28.31) that suggests such a signal.

Recently it has been shown that the nuclear PR becomes cytoplasmic under energy-depleting conditions, demonstrating that a dynamic shuttling occurs with the receptor diffusing out of the nucleus and being actively transported back into the nucleus (Guiochon-Mantel et al., 1991). Besides the ratio of transport in to diffusion out, the balance between binding to nuclear or to cytoplasmic components could also determine the subcellular distribution of steroid receptors. Elements such as the cytoskeleton and heat-shock proteins (hsp90 and hsp70) might keep steroid-hormone receptors cytoplasmic. However, the nuclear matrix and DNA might trap the AR in the nucleus. This could explain the observed hormone-dependent AR nuclear localization in transfected COS-1 cells. Ligand induces the dissociation of the heteromeric complex of receptor with heat-shock proteins, and transforms the receptor into a high-affinity DNA-binding and nuclear-matrix-binding protein (Barrack, 1987). AR13, lacking the steroid-binding domain, is not complexed with hsp90 and constitutively binds DNA (G. Jenster, J. Trapman and A. O. Brinkmann, unpublished work). Therefore, most of these receptors will be present in the nucleus. This finding is in agreement with the proposed model. The compartmental retention model, based upon the dynamic shuttling of steroid receptors, suggests that nuclear import and export are continuous processes and that nuclear import itself is not regulated by ligand binding. This is consistent with the observation that homologous signals are present in many nuclear proteins that are not liganddependent. There are, however, at least two mechanisms by which ligand might indirectly regulate transport of steroid receptors across the nuclear membrane. Firstly, some reports suggest that phosphorylation in or near the NLS could be involved in NLS-nuclear pore interaction and in nuclear import (Rihs and Peters, 1989; DeFranco et al., 1991; Robbins et al., 1991). Hence phosphorylation, possibly induced by ligand, might be a mechanism to regulate the efficiency of nuclear import. Secondly, besides being trapped by binding to cytoplasmic components, cytoplasmic retention could also be caused by masking of the NLS. Hormone-induced changes in structural conformation or the dissociation of the heteromeric complex of the receptor with heat-shock proteins could expose the NLS, resulting in nuclear translocation (Picard and Yamamoto, 1987; Picard et al., 1988; Ylikomi et al., 1992). Evidence for the latter may be provided by the observation that AR17, lacking the last 114 amino-acid residues, was predominantly cytoplasmic, whereas AR14, lacking the last 197 residues, was exclusively nuclear. Possibly, in contrast with AR14, the NLS of AR17 was masked by heat-shock protein association or by conformational interference due to the additional 83 amino-acid residues. Thus, according to the compartmental retention model, at least three 
possible factors could influence subcellular localization: accessibility of the NLS, translocation efficiency, and binding to nuclear or cytoplasmic components. Disruption of the DNAbinding domain did not influence hormone-induced nuclear localization of the AR. Interestingly, clusters of receptors appeared in most nuclei, which were rarely observed in cells expressing the wild type AR. Expression of different AR mutants that were all affected in DNA binding revealed this clustered pattern only in the presence of ligand, indicating that it is unlikely to be a non-specific precipitation. AR21, which lacks the NLS, formed clusters in the cytoplasm.

Some components involved in trapping the AR might be celltype-specific and differently distributed over the cytoplasm and nucleus. In addition, differences in membrane-translocation efficiency could be another explanation for the cell-line-specific distribution of the unliganded AR that was observed in COS-1, CV1, CHO and HeLa cells.

The mechanism of action of steroid-hormone antagonists has been the subject of many studies. There are a number of different processes in the cascade of steroid-receptor activation that may be blocked by antagonists, including the dissociation of the heteromeric complex of the receptor with heat-shock proteins, nuclear import, receptor dimerization, DNA binding and the activation of transcription. For several of these processes there is substantial evidence that they can be blocked by specific antagonists, but our knowledge about the inhibition of nuclear import by antagonists is limited (Kemppainen et al., 1992). Recently it has been shown that the anti-androgen Casodex (ICI 176334) was unable to induce the dissociation of hsp 90, hsp70 and p59 from the AR expressed in cells derived from a lymph node carcinoma of the prostate (Veldscholte et al., 1992). Although this prostatecancer cell line expresses a mutant AR (Thr at position 868 is substituted by ALA; Veldscholte et al., 1990), the experiments with Casodex (ICI 176334) show that the dissociation of the heteromeric hsp-receptor complex is one of the processes that can be blocked by antagonists. To investigate the possibility that anti-androgens and other steroids could interfere with AR nuclear import, different concentrations of R5020, oestradiol, dexamethasone or the anti-androgens hydroxyflutamide, cyproterone acetate and Casodex (ICI 176334) were added to COS-1 cells that were expressing the wild-type AR. All compounds were able to induce nuclear localization, although relatively high concentrations (100 nM) of hydroxyflutamide, Casodex or dexamethasone were necessary. The observation that all these different compounds were able to cause the translocation of the $A R$ to the nucleus suggests that the passage through the nuclear membrane itself is not a ligand-specific process. This is supported by findings that non-ligands such as dopamine and okadaic acid are also able to induce nuclear translocation of the $P R$ and of the GR, respectively (DeFranco et al., 1991; Power et al., 1991). Possibly, upon binding of anti-androgens or other steroids, the AR is retained in the nucleus via an interaction with DNA or with the nuclear matrix. The Casodex-induced nuclear localization of the AR seems to be a more complex process, since there is evidence that Casodex cannot induce the dissociation of the heteromeric hsp-receptor complex. This suggests that the whole complex passes through the nuclear pore, or that disassembly and subsequent re-assembly occur at the nuclear pore. Consequently, DNA is not the nuclear component that traps the hsp-complexed AR. Loss of cytoplasmic retention or interaction with nuclear components such as the nuclear matrix could retain the receptor in the nucleus, similar to AR mutants that are unable to bind DNA. None of the compounds that were tested induced transcriptional activation of an androgen-regulated reporter gene at the lowest concentrations that resulted in AR nuclear localization. This indicates that the inability of the compounds tested to induce transactivation is not caused by an inhibition of nuclear import.

In conclusion, the data presented show that: (1) the NLS responsible for nuclear import of the human AR is encoded by amino-acid residues $608-625$ and is functionally similar to the nucleoplasmin NLS, (2) the ratio of nuclear and cytoplasmic distribution of the unliganded AR is cell-line-specific, (3) disruption of the DNA-binding domain resulted in the formation of AR clusters in the nucleus or in the cytoplasm, and (4) antiandrogens and various steroid hormones induced localization of the AR in a dose-dependent fashion, without affecting transcription activation.

We thank Dr. S. Rusconi for the rat pSTCGR3-795 expression plasmid, Dr. R. Renkawitz for the pG29GtkCAT reporter construct, B. van Steensel and Dr. R. van Driel for helpful interpretations of receptor clustering and Dr. J. A. Grootegoed for heipful discussions and for reading the manuscript. This work was supported by the Netherlands Organization for Scientific Research (NWO) through GB-MW (Medical Sciences).

\section{REFERENCES}

Barrack, E. R. (1987) J. Steroid Biochem. 27, 115-121

Brink, M., Humbel, B. M., de Kloet, E. R. and van Driel, R. (1992) Endocrinology (Baltimore), 130, 3575-3581

Brinkmann, A. O., Faber, P. W., van Rooij, H. C. J., Kuiper, G. G. J. M., Ris, C., Klaassen, P., van der Korput, J. A. G. M., Voorhorst, M. M., van Laar, J. H., Mulder, E. and Trapman, J. (1989) J. Steroid Biochem. 34, 307-310

Chen, C. and Okayama, H. (1987) Mol. Cell. Biol. 7, 2745-2752

DeFranco, D. B., Qi, M., Borror, K. C., Garabedian, M. J. and Brautigan, D. L. (1991) Mol. Endocrinol. 5, 1215-1228

Dingwall, C. and Laskey, R. A. (1991) Trends Biochem. Sci. 16, 478-481

Evans, R. M. (1988) Science 240, 889-895

Green, S. and Chambon, P. (1988) Trends Genet. 4, 309-314

Guiochon-Mantel, A., Loosfelt, H., Lescop, P., Sar, S., Atger, M., Perrot-Applanat, M. and Milgrom, E. (1989) Cell 57, 1147-1154

Guiochon-Mantel, A., Lescop, P., Christin-Maitre, S., Loosfelt, H., Perrot-Aplannat, M. and Milgrom, E. (1991) EMBO J. 10, 3851-3859

Higuchi, R., Krummel, B. and Saiki, R. K. (1988) Nucleic Acids Res. 16, 7351-7367

Husmann, D. A., Wilson, C. M., McPhaul, M. J., Tilley, W. D. and Wilson, J. D. (1990) Endocrinology (Baltimore) 126, 2359-2368

Jenster, G., van der Korput, J. A. G. M., van Vroonhoven, C. C. J., van der Kwast, Th. H., Trapman, T. and Brinkmann, 0. A. (1991) Mol. Endocrinol. 5, 1396-1404

Kalderon, D., Richardson, W. D., Markham, A. T. and Smith, A. E. (1984a) Nature (London) 311, 33-38

Kalderon, D., Roberts, B. L., Richardson, W. D. and Smith, A. E. (1984b) Cell 39, 499-509 Kemppainen, J. A., Lane, M. V., Sar, M. and Wilson, E. M. (1992) J. Biol. Chem. 267. 968-974

Lanford, R. E. and Butel, J. S. (1984) Cell 37, 801-813

Nigg, E. A., Baeuerle, P. A. and Lührmann, R. (1991) Cell 66, 15-22

Perrot-Applanat, M., Logeat, F., Griyer-Picard, M. T. and Milgrom, E. (1985) Endocrinology (Baltimore) 116, 1473-1483

Peters, R. (1986) Biochim. Biophys. Acta 864, 305-359

Picard, D. and Yamamoto, K. R. (1987) EMBO J. 6, 3333-3340

Picard, D., Salser, S. J. and Yamamoto, K. R. (1988) Cell 54, 1073-1080

Power, R. F., Mani, S. K., Codina, J., Conneely, O. M. and O'Malley, B. W. (1991) Science

254, 1636-1639

Rins, H.-P. and Peters, P. (1989) EMBO J. 8, 1479-1484

Robbins, J., Dilworth, S. M., Laskey, R. A. and Dingwall, C. (1991) Cell 64, 615-623

Sanchez, E. R., Hirst, M., Scherrer, L. C., Tang, H.-Y., Welsh, M. J., Harmon, J. M., Simons, S. S., Jr., Ringold, G. M. and Pratt, W. B. (1990) J. Biol. Chem. 265, 20123-20130

Sar, M., Lubahn, D. B., French, F. S. and Wilson, E. M. (1990) Endocrinology (Baltimore), 127, 3180-3186

Schüle, R., Muller, M., Kaltschmidt, C. and Renkawitz, R. (1988) Science 242, 1418-1420 Severne, Y., Wieland, S., Schaffner, W. and Rusconi, S. (1988) EMBO J. 7, 2503-2508 Silver, P. A. (1991) Cell 64, 489-497

Simental, J. A., Sar, M., Lane, M. V., French, F. S. and Wilson, E. M. (1991) J. Biol. Chem. 266, $510-518$

van der Kwast, Th. H., Schalken, J., Ruizeveld de Winter, J. A., van Vroonhoven, C. C. J., Mulder, E., Boersma, W. and Trapman, J. (1991) Int. J. Cancer 48, 189-193 
Veldscholte, J., Ris-Stalpers, C., Kuiper, G. G. J. M., Jenster, G., Berrevoets, C., Claassen, E., van Rooij, H. C. J., Trapman, J., Brinkmann, A. O. and Mulder, E. (1990) Biochem. Biophys. Res. Commun. 173, 534-540

Veldscholte, J., Berrevoets, C. A., Brinkmann, A. 0., Grootegoed, J. A. and Mulder, E. (1992) Biochemistry 31, 2393-2399

Received 2 November 1992/4 February 1993; accepted 12 February 1993
Ylikomi, T., Bocquel, M. T., Berry, M., Gronemeyer, H. and Chambon, P. (1992) EMBO J. 11, 3681-3684

Zegers, N. D., Claassen, E., Neelen, C., Mulder, E., van Laar, J. H., Voorhorst, M. M. Berrevoets, C. A., Brinkmann, A. O., van der Kwast, Th. H., Ruizeveld de Winter, J. A. Trapman, J. and Boersma, W. J. A. (1991) Biochim. Biophys. Acta 1073, 23-32 